Revue européenne des sciences sociales

European Journal of Social Sciences

\title{
Peur de l'inconnu, angoisse et révisions conceptuelles
}

Pierre Livet

\section{(2) OpenEdition}

\section{Journals}

Édition électronique

URL : http://journals.openedition.org/ress/668

DOI : $10.4000 /$ ress.668

ISSN : 1663-4446

Éditeur

Librairie Droz

Édition imprimée

Date de publication : 1 décembre 2000

Pagination : 45-64

ISBN : 2-600-00481-5

ISSN : 0048-8046

Référence électronique

Pierre Livet, « Peur de l'inconnu, angoisse et révisions conceptuelles », Revue européenne des sciences sociales [En ligne], XXXVIII-119 | 2000, mis en ligne le 15 décembre 2009, consulté le 01 mai 2019.

URL : http://journals.openedition.org/ress/668 ; DOI : 10.4000/ress.668 
Pierre LIVET

\section{PEUR DE L'INCONNU, ANGOISSE ET RÉVISIONS CONCEPTUELLES}

Jean-Claude Passeron nous a parlé de l'importance des affects propres à l'activité intellectuelle. Je voudrais explorer quelques mécanismes des ces affects, les pièges qu'ils nous tendent, mais aussi montrer leurs avantages, qui sont plus facilement évalués au niveau collectif. Je citerai la peur de l'inconnu, l'angoisse d'avoir à sortir de son cadre de recherche, l'attrait de l'inconnu, l'attirance pour la multiplication des efforts, et la satisfaction quasi maligne d'obtenir avec peu de changements conceptuels une extension importante des résultats scientifiques (curieusement, les deux derniers peuvent se combiner, quand on multiplie les calculs et les expériences sans changer de concepts). Il me semble que tous ces affects sont liés entre eux par leurs relations avec un processus essentiel à la recherche scientifique (mais aussi à nos recherches morales et pratiques), celui de la révision, qui nous amène à changer de théorie ou d'hypothèses quand nous nous révélons en contradiction avec les faits.

Plusieurs questions se posent à nous. Tout d'abord, est-ce que des êtres qui ne seraient pas dotés d'émotions seraient soumis aux mêmes obstacles dans leur recherche que nos intellectuels encombrés d'affects? Quand Passeron parle de point aveugle dans une théorie, il suppose que c'est là une propriété de toute théorie, et qui ne tient pas essentiellement aux dispositions affectives de ses auteurs, ni même à leur psychologie épistémique, mais bien à la disposition structurelle de chaque théorie. Il ne s'agit pas pour lui d'une sorte de duperie de soi, l'intellectuel étant incapable de voir ce qui contredit sa théorie parce que cela lui ferait trop de peine, ni d'une sorte de cécité épistémique, tenant à ce que le concepteur d'une théorie a dû se focaliser sur certains aspects du monde plutôt que sur d'autres. Sa position, semble-t-il, va au delà. Une théorie exige de restructurer les catégories entre elles, et cette restructuration même exclut d'autres structurations, si bien que toute théorie rend impossible l'accès à une multitude d'autres théories possibles, chacune en accord avec des faits autrement découpés. Pour lui, la peur de ce point aveugle est alors le seul indice psychologique qui reste au chercheur de cette conscience latente qu'il s'est coupé d'autres organisations conceptuelles. A vrai dire, il s'agit plutôt d'une angoisse, si on reprend le cliché conceptuel qui veut que la peur ait un objet précis tandis que l'angoisse n'en ait point. Or l'inconnu ou le point aveugle peuvent difficilement être tenus pour des objets précis. L'existence du second nous empêche en effet d'explorer le premier, et le second ne peut être déterminé qu'imparfaitement, puisque s'il est un obstacle à la découverte d'autres théories, une fois ces théories rendues accessibles, il ne sera pas davantage définissable pour autant. 
La thèse que nous défendrons est proche de celle de Passeron, mais en léger décalage. Si les théories sont des manières d'explorer les relations stables dans nos réalités physiques et culturelles, alors un être qui n'aurait pas d'émotions et surtout qui n'aurait pas cette émotion qu'est l'angoisse de l'inconnu (mais aussi son attrait) ne disposerait pas des motivations nécessaires pour se lancer dans des explorations dès lors qu'il n'entreverrait pas au présent de raisons suffisamment décisives de le faire. Même si l'angoisse de l'inconnu est une émotion à double face qui peut tout aussi bien nous engager dans l'exploration que nous en décourager, elle finira toujours par permettre plus d'explorations qu'un être sans émotions n'en aurait entreprises. Et la raison de ce dynamisme se trouve dans la liaison qui existe entre émotions et révision. Cependant, nous le verrons, l'avantage que donne les émotions ne peut s'observer qu'au niveau d'une population de chercheurs, et non pas au niveau individuel.

Réviser, c'est d'abord observer une contradiction entre les faits et ce que nos croyances et hypothèses nous faisaient attendre comme données. A supposer que nos inférences aient été correctes, il faut savoir quelles croyances éliminer parmi celles qui ont servi à produire notre attente erronée. Quel peut alors être notre guide pour choisir ce qu'il faut abandonner? On peut vouloir minimiser l'extension de la révision. Mais il est bien possible que plusieurs ensembles de croyances d'extension similaires soient candidats à la révision. Il faut donc choisir entre eux. On va devoir alors le faire en tâchant de conserver les croyances qui sont pour nous prioritaires. On peut d'ailleurs, avec plus de plausibilité et de réalisme, prendre les choses dans l'autre sens. Le choix des croyances ou des hypothèses que nous éliminons en cas de contradiction avec les faits révèle quelles sont nos priorités.

Mais cette révision partielle ne se heurte-t-elle pas au holisme de DuhemQuine, qui soutient qu'un fait en contradiction avec les prévisions d'une théorie attaque la théorie dans son ensemble, et non pas simplement une des parties de la théorie? On se trouverait alors pris dans un dilemme. Soit on admettrait des procédures de révision, mais il faudrait alors pouvoir récuser le holisme et montrer que certaines parties des théories sont indépendantes les unes des autres, soit on tiendrait pour acquis le holisme de la signification défendu par Quine, et selon lequel un concept ne prend sens que dans un réseau de concepts, mais alors il faudrait renoncer non pas à la réfutation des théories, mais à la possibilité de réfuter une partie d'une théorie pour la réformer. Cependant, si nous sommes holistes, notre comportement scientifique révèle que nous avons des priorités épistémiques. Que nous reconstruisions le radeau du savoir en restant à son bord et en enlevant des planches ici pour les mettre ailleurs ne nous empêche donc pas de révéler nos priorités en manière de révision. Davantage, l'idée que la révision d'une théorie se fait en tenant compte des priorités épistémiques de cette théorie est un moyen de limiter l'extension de la révision tout en tenant compte de son statut holistique. Le système de priorités est en effet le résultat de la théorie toute entière, mais au lieu que ce rapport à la théorie dans son ensemble rende impossible l'élimination d'hypothèses isolées, le système des priorités permet au contraire de choisir les hypothèses qu'il est préférable de sacrifier si l'on veut précisément conserver au maximum le réseau de concepts propre à la théorie. Ce processus de révision se présente donc comme une solution au dilemme du holisme et de la réfutation. 
Si la recherche scientifique a des priorités épistémiques, ces priorités ne sont pas entièrement neutres affectivement. Préférer le simple au complexe, l'élégance à la lourdeur, ce sont là des priorités esthétiques, et qui peuvent donner lieu à des affects. Cependant ne pourrait-on imaginer un être cognitif qui n'aurait pas d'émotions, alors même qu'il disposerait de priorités épistémiques? Il pourrait même disposer de priorités esthético-épistémiques, si du moins il existait quelques critères rationnels de la simplicité ou de l'élégance d'une théorie. Un tel être pourrait réaliser toutes les révisions nécessaires. Que nous apportent alors les émotions dans le domaine de la connaissance, et quel rôle peut jouer l'angoisse dans ce jeu entre émotions et connaissance?

Comment se comporterait un être sans émotions? Supposons qu'une théorie ne rencontre pas pour l'instant de contre-exemple. Cet être pourrait cependant se lancer dans l'exploration d'autres théories, dans la mesure où il tiendrait compte de l'histoire passée des sciences, qui lui a montré qu'aucune théorie n'est pleinement satisfaisante ni suffisante. Mais il devrait estimer l'espérance d'utilité d'une telle exploration, qui a tout de même un coût. Ce coût tient à ce que notre être sans émotion doit tout de même dépenser des efforts cognitifs à la mesure de la complexité des problèmes qu'il traite. Un tel être ne se lancerait donc dans une exploration cognitive que s'il pouvait estimer d'avance que la probabilité de rencontrer dans le domaine à explorer des découvertes intéressantes l'emporterait sur le coût de l'exploration. Autrement dit, une fois lancé dans une exploration, s'il se révélait qu'elle dure plus longtemps que prévu sans donner de résultats, notre être sans émotions devrait prendre la décision de l'abandonner. Mais il est aussi une deuxième tâche cognitive, duale de l'exploration de nouvelles théories, c'est la défense des théories déjà convaincantes. Là aussi, notre être rationnel mais sans émotions calculerait l'espérance d'utilité de cette défense, le gain consistant dans le maintien d'une théorie intéressante, au lieu de l'abandonner trop vite à la moindre difficulté, le coût consistant dans la complexité des manoeuvres nécessaires pour restaurer la théorie entamée par des attaques successives. Là encore, une fois que le seuil estimé serait dépassé, il devrait abandonner cette défense. Notons que si nous avions affaire à une population de tels êtres non émotionnels, ils ne pourraient différer dans leur estimations qu'en fonction de différences dans leurs informations. Mais il en serait comme sur un marché de concurrence parfaite. Même sans se communiquer leurs informations, leur comportement et leurs décisions révéleraient quel impact auraient ces informations sur leur décisions, si bien que ces êtres rationnels ajusteraient peu à peu leurs estimations jusqu'à ne former qu'un seul collectif homogène. Finalement, la population toute entière prendrait les mêmes décisions d'arrêt de l'exploration d'une nouvelle théorie ou de la défense d'une ancienne théorie.

Il nous faut maintenant montrer le lien entre émotions et révision. Définissons une émotion comme la réaction affective et physiologique à la perception ou en général la prise de connaissance, voire l'imagination, d'un différentiel entre nos orientations et activités en cours et la situation actuelle ou imaginée. Un différentiel tient à une modification qui impose une inflexion voire une discontinuité estimée en fonction de nos préférences et de nos dispositions à l'action actuelles. Une situation qui est source d'émotion impose donc un premier type de révision: nous découvrons que la situation est différente du simple prolongement de nos percep- 
tions et activités en cours. Concevons ces perceptions comme des anticipations. Nos anticipations avaient alors une conclusion que nous devons modifier en fonction de la nouvelle situation. C'est là une révision minimale. Elle ne porte que sur la conclusion de notre attente, elle ne remet pas en cause ses prémisses, ni le processus qui va des prémisses à la conclusion. Comment est-ce possible? La majeure partie de nos attentes sont des attentes «normales», si les choses se passent comme d'habitude. Cela n'implique nullement qu'elles se passent toujours comme cela. Si bien que nos attentes comportent en elles-mêmes la possibilité d'exceptions. Quand une telle exception se produit, nous ne renonçons donc pas aux bases de nos attentes, puisqu'elles ne valaient que sauf exception, normalement et dans la plupart des cas. Nous ne remettons pas non plus en question les processus qui nous portent à ces conclusions, parce que la situation exceptionnelle n'a pas beaucoup de chances de se reproduire.

En revanche, supposons que la situation que nous pensions exceptionnelle se reproduise assez fréquemment. Alors l'émotion renaît sans cesse. Notre hypothèse est qu'alors, l'émotion nous motive pour un processus de révision, qui, s'il est mené à bien, va changer nos attentes, si bien que peu à peu la situation en cause, lorsqu'elle se reproduit, n'entraîne plus qu'une émotion de plus en plus atténuée. L'émotion diminue voire disparaît par accommodation quand la révision est accomplie. Nous savons que pour éviter l'émotion, il ne suffit pas détourner les yeux de la réalité et de nous cacher à quel point elle diffère de nos attentes. Car sinon, l'émotion réapparaîtra. L'émotion est sur ce point plus proche de la perception que de nos désirs. Elle nous rappelle que le monde est soit plus défavorable à nos attentes que prévu, soit, pour les émotions positives, plus favorable.

On peut noter que toutes les émotions n'ont pas ce destin. Nous nous accommodons difficilement à une violence continuelle, sauf si nous sommes nés dans un environnement violent, nous nous accommodons même difficilement à une absence presque continuelle de soleil, à moins là aussi d'y être habitué dès la naissance, etc. Par ailleurs, le mode d'accommodation diffère entre les émotions positives et les émotions négatives. Nous nous accommodons moins aux situations positives, et cela nous permet de conserver la capacité de ressentir des émotions positives alors même que la situation heureuse devient plus fréquente, bien que là aussi, une répétition trop suivie mène à l'ennui. Nous nous accommodons davantage à la plupart des émotions négatives. Le fait de ne pas pouvoir nous accommoder à certaine situations nous révèle quelles sont nos préférences les plus fermement enracinées.

Nous pouvons accroître cette résistance en partageant nos émotions. Vérifier que nos congénères eux aussi persistent à s'attendre à ce que le monde soit plus favorable à certaines attentes qu'il ne l'est en moyenne, et donc ressentent eux aussi des regrets, des insatisfactions ou des indignations, c'est ce que nous permet le partage, qui nous renforce donc dans notre résistance.

Il faut enfin signaler un autre trait de la dynamique et de l'évolution à long terme des émotions. Comme l'émotion est liée à la perception d'un différentiel avec nos attentes, à supposer que nous nous engagions dans une entreprise dont la réussite ne nous semble pas assurée, nous allons d'abord, puisque nos attentes sont plutôt négatives, être hypersensibles aux indices de succès. Puis, une fois que nous nous sommes accoutumés et que nos attentes sont devenues plus optimistes, le différentiel va s'inverser, et nous deviendrons donc plus sensibles aux indices 
d'échec. Ce serait seulement sur le très long terme que nous pourrions trouver un équilibre, et en fait nous serons probablement entraînés dans une succession d'oscillations entre ces deux types de phase.

Revenons maintenant à notre être cognitif, et dotons-le d'émotions. Sa théorie détermine ses attentes. Il devient donc plus sensible aux différentiels avec ces attentes qu'il ne l'était quand il n'avait pas d'émotions. Cela lui donne deux types de motivation. D'une part, la répétition de tels différentiels, qui provoquent des émotions négatives, l'incite à s'engager dans une révision de ses attentes, donc de sa théorie. L'émotion, ici, ne joue que le rôle d'une sorte d'alarme, qui résonne chaque fois qu'on perçoit le différentiel. La répétition de l'alarme suffit cependant à souhaiter éviter son retour. Et l'on ne peut espérer éviter le retour de l'alarme en détournant ailleurs nos préocuppations, puisque l'émotion est comme la perception, elle se produit que nous le voulions ou pas. Cependant, notre être émotionnel s'aperçoit que malgré les tentatives de révision dans lesquelles il s'engage poussé par la répétition de cette alarme, il est certaines prémisses qu'il ne révise pas, alors même qu'elles le conduisent toujours à des conclusions en distorsion avec les situations qu'il rencontre. Il dispose ainsi d'un moyen de se révéler à lui-même ce qui compte vraiment pour lui dans sa théorie et ce à quoi il est moins attaché. Un être qui a des émotions a donc d'une part une plus forte tendance à réviser certaines prémisses et croyances, d'autre part une plus forte tendance à résister à la révision d'autres croyances. Il se révèle plus vite à lui-même les différences de priorités entre les différents éléments de la théorie. Il peut donc plus facilement disposer d'un guide dans son entreprise de révision. Certes, nous pourrions supposer notre être sans émotions doté d'une parfaite connaissance des priorités dans sa théorie. Mais il suffit que la découverte de ces priorités soit une tâche difficile pour que les émotions puissent nous servir.

Assurément, notre théoricien ému pourrait résister à la révision pour des motifs qui tiennent à ses désirs et non pas à ses croyances, par exemple parce qu'il est l'auteur de telle thèse maintenant contestée. Mais souvenons-nous que nous considérons une population de tels théoriciens émus. En effet, si les comportements de résistance ou de persistance dans l'exploration peuvent être attribués à des émotions qui pourraient rester personnelles, les autres chercheurs, quand au départ ils n'éprouvent pas les mêmes émotions, n'ont pas forcément de raison de converger avec ceux qu'ils voient attachés à la résistance d'une théorie, ou au contraire persévérant dans des explorations infructueuses. Une telle population montrera donc plus de dispositions d'une part à réviser, d'autre part à résister aux révisions. Une telle population, surtout, conservera son hétérogénéité. On pourrait cependant objecter qu'une force joue dans le sens de l'homogénéisation, c'est la tendance des êtres émotionnels à partager leurs émotions. On l'a vu, le partage des émotions nous porte à résister à la réalité quand elle ne correspond pas à nos attentes, et une population de théoriciens émus pourrait donc rester très conservatrice.

Mais ici le partage des émotions ne joue pas forcément dans le sens de l'homogénéisation. Il permet à des gens qui résistent à la réalité de se sentir confortés parce qu'ils partagent la même résistance. Mais cela permet aussi bien aux conservateurs de se sentir confortés entre eux qu'aux explorateurs de ne pas se résigner à abandonner leur exploration. D'une part, ce partage des émotions n'est pas un 
mécanisme psychologique qui fonctionne à grande distance, comme le marché. $\mathrm{Au}$ contraire, il exige une familiarité entre les personnes qui partagent leurs émotions, et il est plus efficace dans des petits groupes. D'autre part, on peut partager aussi bien le refus de certaines expériences gênantes que l'obstination dans une recherche qui pour l'instant n'offre que des promesses et pas de résultats probants. Si bien que même le partage, dans le domaine émotionnel, renforce l'hétérogénéité. Les émotions ont donc pour effet, dans une population de chercheurs, d'accentuer les différences individuelles entre ceux qui résistent et ceux qui explorent, en renforçant les relations à l'intérieur de petits groupes, mais en accentuant aussi les différences entre ces groupes. Les émotions réinjectent donc de l'hétérogénéité dans la population de chercheurs, et permettent aux chercheurs à la fois de dépenser plus d'énergie à résister ou à explorer qu'ils ne le feraient s'ils étaient sans émotions. Au total, une telle population a plus de chances d'explorer de nouvelles théories, et de consacrer assez d'énergie à la défense des anciennes contre des réfutations qui pourraient se révéler apparentes.

Enfin une population conservatrice, si l'on considère les phases de la dynamique des émotions, reste bloquée dans une phase d'hypersensibilité aux indices négatifs en défaveur de la théorie qu'elle tient à conserver (pour passer dans l'autre phase, il faudrait qu'elle devienne d'abord persuadée que sa théorie a toute chance d'être dégénérative!). Elle se trouve donc dans une situation difficile, qui tient à ce que des émotions négatives l'assaillent sans cesse à cause de cette hypersensibilité, à ce qu'elle voit de moins en moins de moyens de réajuster sa théorie de manière satisfaisante, et que, fixée sur sa théorie favorite qui lui apparaît le seul salut, elle ne voit pas comment en sortir pour la réviser par une autre théorie.

Nous rencontrons alors l'émotion dont nous sommes partis, à savoir l'angoisse. Nous pouvons en effet définir l'angoisse comme l'émotion que nous éprouvons quand à la fois nous ressentons la nécessité d'une révision et que nous ne voyons pas comment l'accomplir, pour des raisons diverses, soit que nous n'ayons pas de pistes de recherche, soit que ces pistes nous conduisent à mettre en question des attentes qui sont pour nous de priorité fondamentale. Nos théoriciens conservateurs sont donc forcément aussi angoissés.

L'angoisse produit en nous différentes motivations. D'une part elle nous signale l'urgence d'une révision, d'autre part elle nous signale son importance. Ces motivations peuvent aller en sens inverse l'une de l'autre, une révision importante étant difficile à faire puisqu'il faut bouleverser notre organisation conceptuelle, ce qui nous incite à ne pas nous lancer dans cette entreprise délicate, une révision urgente exigeant d'être aussitôt engagée. Nous sommes d'autant plus angoissés que les priorités qui peuvent être mises en question dans la révision risquent d'être plus fondamentales. Là encore, comme toutes les émotions négatives, l'angoisse joue bien le rôle d'un signal d'alarme. Ce nous est une motivation à trouver des moyen d'éviter sa réoccurrence. Mais comme l'angoisse nous signale aussi que la révision est fondamentale, et que nous ne disposons pas de guide pour la faire, nous nous doutons qu'elle sera difficile. En effet, pour accomplir une révision, nous devons éliminer nos attentes qui sont les moins prioritaires. Or l'angoisse nous indique à la fois que des attentes très prioritaires peuvent être en cause, et que nous ne savons pas pour le moment comment minimiser la révision et découvrir des attentes moins prioritaires qui permettraient de réviser. Ce qui revient à dire que nous n'avons pas de guide pour minimiser la révision. 
Si donc nous avons la possibilité d'entreprendre une révision de dérivation, qui nous semble plus minime que la révision qui provoque l'angoisse, mais qui nous permet cependant de nous focaliser sur une tâche de révision, nous allons être tenté de nous y plonger. On voit ainsi des scientifiques refaire plusieurs fois l'expérience qui ne correspondait pas à leurs attentes, et on peut aussi interpréter de cette manière la construction de théories «épicycliques», qui pour chaque objection développent une complication de la théorie, complication qui est donc ad hoc, et permet d'éviter de remettre en cause les thèses centrales de la théorie. Mais la construction de tels appendices théoriques ad hoc ne peut nous soulager de l'angoisse que temporairement. La révision fondamentale reste à faire, et l'angoisse redémarre sans cesse. L'angoisse reste donc une pression pour une révision plus profonde alors même qu'elle peut toujours nous amener à nous jeter dans des révisions de dérivation.

Si on revient au problème de l'exploration de la réalité (ou du domaine des concepts) par les théories, exploration menée par une population de chercheurs, on voit que l'angoisse donne une motivation pour mener l'exploration beaucoup plus loin que ne le ferait un chercheur sans émotions, parce qu'elle nous amène à envisager la remise en question des bases fondamentales d'une théorie au lieu de nous borner à renforcer ce que Lakatos appelle sa ceinture de protection. Mais elle nous amène aussi à tenter toutes les actions de résistance à ces révisions fondamentales qui sont à notre portée. Il faut donc que la population de chercheurs soit assez importante et diversifiée pour que l'angoisse soit bénéfique, puisqu'alors pour toute résistance on pourra trouver d'autres chercheurs qui seront poussés à aller très loin dans l'exploration.

Le bilan de l'angoisse en termes de recherche semble être le suivant. D'une part, l'angoisse nous rappelle qu'il faut parfois procéder à des révisions fondamentales au lieu de se contenter de révisions minimales et périphériques. D'autre part, la même angoisse nous pousse à nous précipiter dans ces révisions périphériques, justement pour lui échapper temporairement (nous n'avons cependant pas besoin d'avoir conscience que nous entreprenons ces révisions locales à cette fin, il nous suffit de les entreprendre, et ce choix est alors renforcé par le fait qu'il apaise temporairement l'angoisse; aucun finalisme n'est ici nécessaire). Mais il faut tenir compte d'autres phénomènes. D'une part, le partage de l'angoisse l'accentue au lieu de la calmer. D'autre part, la résistance d'un groupe à une remise en cause de sa théorie, une fois qu'elle peut être interprétée comme un signe d'angoisse - même déniée par ces conservateurs - est une raison de persévérer pour les tenants d'explorations nouvelles. Et les révisions de dérivation, ou encore tout simplement le refus de toute modification, le dogmatisme, sont pour ceux qui souhaitent attaquer la théorie des signes d'angoisse. Enfin, le fait que nous n'ayons pas de guide pour minimiser la révision nous indique que le cadre même de notre théorie peut être remis en jeu. L'angoisse jette le doute sur les priorités jusque là admises. Les conservateurs angoissés voient donc s'élargir le fossé entre des révisions locales qui ne portent que sur des thèses non prioritaires dans la zone de la ceinture de protection, et des révisions plus fondamentales, mais dont on ne voit pas comment les faire. La théorie devient hétérogène, et ne sert plus de guide cohérent. L'angoisse permet donc que la fermeture même d'un groupe sur luimême donne des armes et de l'énergie à un groupe externe ou hétérodoxe. Elle 
amène aussi une sorte de dislocation interne des priorités qui servent de guide dans l'évolution et le perfectionnement de la théorie.

L'angoisse avive ce qu'on pourrait appeler les pathologies de la recherche: la dépense d'efforts considérables pour des révisions de dérivation qui ne font que reculer les problèmes, le dogmatisme qui refuse la discussion, le blocage sur les positions d'une théorie, et elle exaspère les conflits entre les chercheurs conservateurs et les autres, mais elle donne aussi des raisons d'espérer à ceux qui veulent mettre en question la théorie dominante et qui auraient pu être découragés par le peu d'effet de leurs explorations. Ceux-là peuvent partager leur résistance à la théorie en cours et bénéficier de l'effet de renforcement propre au partage des émotions entre familiers. Leur problème était qu'ils n'avaient pas au départ de guide bien clair dans leurs explorations et leurs tentatives de révision. Ils savaient simplement qu'ils ne pouvaient se satisfaire d'une révision périphérique, et ils attaquent donc ce qu'ils ressentent comme l'une des bases de la théorie en cours. Cela même leur sert de guide, mais ne leur donne aucune assurance qu'ils découvriront de nouveaux principes qui permettent de faire dees découvertes intéressantes. Mais maintenant l'angoisse (et surtout l'angoisse des autres) devient un moteur de recherche, parce que s'attaquer à ce qui est le plus angoissant à mettre en question, c'est utiliser le signal d'alarme de l'angoisse comme un fanal qui indique où chercher. Et nos hétérodoxes peuvent alors affronter l'idée que ce n'est pas là une garantie suffisante de succès dans leur exploration, parce qu'ils ont la certitude d'avoir attaqué une priorité fondamentale. Si l'on imagine l'espace de la recherche comme un paysage avec des bassins, des crêtes et des cols, remettre en cause des priorités de base, c'est devoir aller chercher très loin et franchir beaucoup de cols parce que l'on veut trouver un bassin plus profond que le bassin de départ. Nos empêcheurs de penser en rond s'appuient sur cette certitude pour penser que leur recherche est prioritaire et, sachant qu'elle exige une remise en cause fondamentale, ils s'attendent à ne pas pouvoir obtenir des résultats satisfaisants avant d'avoir dépensé beaucoup d'efforts et ne sont plus autant déçus pas leurs échecs successifs.

L'angoisse renforce donc trois types de comportement: la recherche de révisions locales, qui est dans la plupart des cas une perte de temps, le blocage sur les positions théoriques initiales, qui ne fait évidemment pas avancer les connaissances, mais elle donne aussi des raisons de persévérer aux chercheurs hétérodoxes qui peuvent reporter leur angoisse sur les épaules des autres, et ne conserver pour eux-mêmes l'angoisse que comme signal de la fondamentalité de leur recherche. Et elle mine la cohérence interne de la théorie en cours, en accentuant la distance entre l'attachement désespéré aux fondements et la dépense en efforts périphériques dont les spécificités sont de plus en plus compliquées à relier aux bases de la théorie. Au total, l'angoisse est une source de conflits entre des positions rigidifiées et des hétérodoxies plus assurées de leur bon droit, et elle conduit les dogmatismes rigides à un affaiblissement interne de leurs positions. Cependant le conflit va resserrer le débat intellectuel autour de quelques points focaux, ce qui est sans doute un obstacle à la poursuite de recherches plus étendues. Mais si la rigidification a tendance à retarder l'évolution intellectuelle, en même temps elle amène les théories dogmatiques à se miner elles-mêmes, si bien qu'au total l'évolution n'est peut-être pas retardée, mais simplement rendue plus discontinue, ce qui donne les effets de paradigme notés par Kuhn. Le résultat le plus clair de 
l'angoisse est de renforcer les énergies des explorateurs en conflit direct et fondamental avec la théorie en cours. Si bien que finalement, dans une population de chercheurs - et non chez un seul individu - l'angoisse donne l'énergie de remettre en cause les fondements d'une théorie alors même que cette théorie ne rencontre pas de contre-exemple décisif. Là encore, l'émotion est finalement un moteur pour explorer non seulement un domaine plus étendu, mais un domaine plus profond. On voit donc que si les émotions elles peuvent diminuer les capacités de recherche des individus en les engageant dans des conduites qui à leur échelle sont irrationnelles, elles augmentent les capacités de recherche et d'exploration d'une population.

Il existe d'autres émotions intellectuelles. En particulier, l'inconnu ne nous angoisse pas toujours. Si nous pouvons penser qu'une solution est proche et ne nécessite qu'une faible exploration, cette perspective ne va pas nous angoisser mais au contraire nous attirer et nous exciter. Mais cette excitation peut être fallacieuse, si par exemple nous nous imaginons qu'il suffit de nous lancer dans l'hétérodoxie pour découvrir aussitôt la solution des problèmes laissés de côté par les options fondamentales des théories précédentes. Si nous attaquons les options fondamentales d'une théorie bien installée, nous devons nous attendre à avoir à faire un travail très lourd et à ce que l'exploration demande beaucoup d'efforts. Il nous est plus aisé de penser qu'il suffit de changer de priorités pour que tout s'éclaire. L'argument à l'appui de cet espoir est que les difficultés rencontrées tenaient à des priorités mal ordonnées, et qu'un changement de priorités, tout en impliquant une révolution émotionnelle, peut nous épargner un travail d'exploration important, en nous faisant pour ainsi dire sauter dans une région lointaine du paysage de la recherche sans avoir eu à explorer les régions intermédiaires. Cette idée est attirante, car elle transforme une révision difficile en révision minimale. Mais elle est fallacieuse, parce que si on faisait ce saut on ne saurait pas comment raccorder les deux régions de savoir, et que notre connaissance resterait incohérente, donc sans guide stable pour entreprendre nos révisions.

En conclusion, dire que tout système intellectuel comporte un point aveugle et que les comportements intellectuels des chercheurs par rapport à ce point aveugle se manifestent par des conduites émotionnelles, qui révèlent de l'angoisse, c'est finalement dire que toute théorie comporte un système de priorités, et que l'exploration intellectuelle exige parfois de changer de priorités, si bien qu'entre les deux systèmes un hiatus doit être franchi, hiatus qu'on ne peut définir puisqu'on est obligé de parler depuis l'une ou l'autre des théories, et pas entre les deux. A ce hiatus correspond effectivement l'émotion de l'angoisse, qui peut être vécue directement par les chercheurs, qui peut se manifester indirectement par leurs conduites de blocage dogmatique, ou de concentration sur des révisions superficielles, ou enfin qui peut servir de moteur à des chercheurs hétérodoxes, dans la mesure où l'angoisse des orthodoxes leur sert de soutien et de confirmation de la pertinence de leur entreprise.

On peut aussi interpréter dans cette problématique l'angoisse et le raidissement dogmatique qui saisit les juristes positivistes face aux écoles qui donnent une grande part à l'interprétation des juges (cf. l'intervention de Timsit ci-dessous). Mais on peut alors se demander si le pari pour la complexité de 
l'interprétation peut effectivement résoudre tous les problèmes qui restent pendant dans une théorie positiviste. En revanche la peur d'un enfermement autoréférentiel du droit, signalée par Jouanjan, est une angoisse que seuls peuvent ressentir ceux qui n'ont ni vraiment choisi le camp de la théorie positiviste et de son dogmatisme, ni celui des interprétationnistes avec leurs difficultés à développer des théories cohérentes entre elles. Car cette angoisse ne peut pas être ressentie du côté dogmatique, qui a déjà ses principes de clôture, ni du côté des interprétationnistes, puisqu'ils y verraient une source de confirmation de leurs efforts. Elle ne peut donc être ressentie qu'au niveau d'une description méta-théorique du domaine de la recherche en théorie du droit. Les émotions peuvent donc jouer jusque dans les descriptions méta-théoriques.

Université d'Aix-Marseille 